\title{
Reconstruction of Scattered Data in Fetal Diffusion MRI
}

\author{
Estanislao Oubel ${ }^{1}$, Meriam Koob $^{2}$, Colin Studholme ${ }^{3}$, Jean-Louis Dietemann ${ }^{2}$, \\ and François Rousseau ${ }^{1}$ \\ 1 LSIIT, UMR 7005, CNRS-Université de Strasbourg, France \\ 2 LINC, UMR 7191, CNRS-Université de Strasbourg, France \\ 3 UCSF, Radiology Department, San Francisco, CA 94143
}

\begin{abstract}
In this paper we present a method for reconstructing D-MRI data on regular grids from sparse data without assuming specific diffusion models. This is particularly important when studying the fetal brain in utero, since registration methods applied for movement and distortion correction produce scattered data in spatial and angular (gradient) domains. We propose the use of a groupwise registration method, and a dual spatio-angular interpolation by using radial basis functions (RBF). Experiments performed on adult data showed a high accuracy of the method when estimating diffusion images in unavailable directions. The application to fetal data showed an improvement in the quality of the sequences according to criteria based on fractional anisotropy (FA) maps, and differences in the tractography results.
\end{abstract}

\section{Introduction}

Diffusion Magnetic Resonance Imaging (D-MRI) is an imaging modality increasingly used for studying the normal and pathological development of the fetal brain. Since the limited sensitivity of fetal ultrasound to detect and depict the maturational processes of the developing white matter, D-MRI can be considered as one of the most promising methods for studying in vivo the human white matter development.

Eddy current-induced image distortions and patient motion during prolonged acquisitions cause image misalignment in D-MRI sequences, invalidating the assumption of a consistent relationship between image space and anatomy in the image processing. Such distortions are generally assumed to be affine, and usually corrected by coregistration with the $T_{2}$-weighted image $\left(T_{2}^{e p i}\right)$ by using affine transformation models and mutual information (MI) [1. When imaging a fetus, there exists the additional problem of movement, and the acquired slices are no longer a regular sample of the volume [2] 3. This may be compensated by relaxing the 3D transformation model to a set of transformations applied to each slice independently. When these transformations are applied to the original sequence, scattered data are generated in the spatial and angular (gradient) domains, requiring an interpolation in both fields. Recently, Jiang et al. 3] have presented

T. Jiang et al. (Eds.): MICCAI 2010, Part I, LNCS 6361, pp. 574 581 2010

(C) Springer-Verlag Berlin Heidelberg 2010 
an interpolation method assuming that the local diffusion properties can be represented by a rank-2 tensor model. However, this model cannot describe voxels containing multiple fibers with different orientations, a condition referred to as intravoxel orientational heterogeneity (IVOH) 4.

In this paper, we present a reconstruction method independent of the diffusion model than can be used with more complex diffusion models like Gaussian mixture models or higher-order diffusion tensors. Distortion corrections are performed by applying groupwise registration with an affine slice-by-slice transformation model. A dual RBF-based interpolation in the spatial and angular domains was then used for reconstruction on a regularly sampled grid.

\section{Method}

\subsection{Origin of Sparse Data}

Typically, a D-MRI sequence consists of a set of $N+1$ regularly sampled images $\mathcal{S}=\left\{S_{0}, S_{1}, \cdots S_{N}\right\}$ where $S_{0}$ is the image obtained without diffusion weighting, and $S_{i=1: N}$ are diffusion-weighted (DW) images obtained with diffusionsensitizing gradients $G_{i}$ of direction $U_{i}$ and strength $b$. Ideally, $S_{0}$ and $S_{i}$ are related by the Stejskal-Tanner equation, but distortions caused by eddy currents and fetal motion invalidate this relationship. Image registration techniques can be applied to restore the lost spatial correspondence between $S_{0}$ and $S_{i}$, but a reconstruction from sparse data is then required.

Let us express the original sequence as $\mathcal{S}=\left\{(X, \Theta, S(X, \Theta)\}_{X \in \Omega_{s}, \Theta \in \Omega_{a}}\right.$ where $\Omega_{s}$ is a regular spatial grid, and $\Omega_{a}$ is a regular angular grid on the unit sphere.

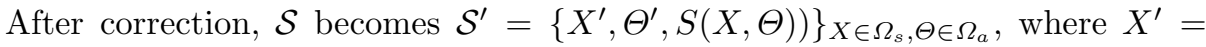
$A_{i}^{z}(X)$ are the transformed spatial points, and $\Theta^{\prime}=R_{i}^{z}(\Theta)$ are the coordinates of $U_{i}$ corrected with the rotational component $R_{i}^{z}$ of $A_{i}^{z}$. In these equations, the superscript " $z$ " has been added to indicate the slice-to-slice nature of the transformations. Differently of $(X, \Theta)$, the transformed coordinates $\left(X^{\prime}, \Theta^{\prime}\right)$ do not belong to regular grids, and $\mathcal{S}^{\prime}$ results sparse.

\subsection{Registration Approach}

Distortion correction methods relying on the registration of $S_{i}$ to $S_{0}$ may fail when applied to fetal D-MRI, probably a consequence of the large differences between both images [3. In this paper, we propose a method that takes advantage of the similarity between DW images to first ensure their joint alignment. The mean of the registered images is characterized by a higher SNR than images $S_{i}$, and provides a better depiction of the anatomical structure of the brain. These properties allow an accurate registration to $S_{0}$, necessary to map all the sequence in its space of coordinates. This method is illustrated and explained in Figure 1 . In essence, the method is similar to the one propose by Guimond et al. [5]. The main difference is the separation of the registration into (1) a groupwise registration of the DW images, and (2) the registration of the resulting mean image with the anatomical image. 


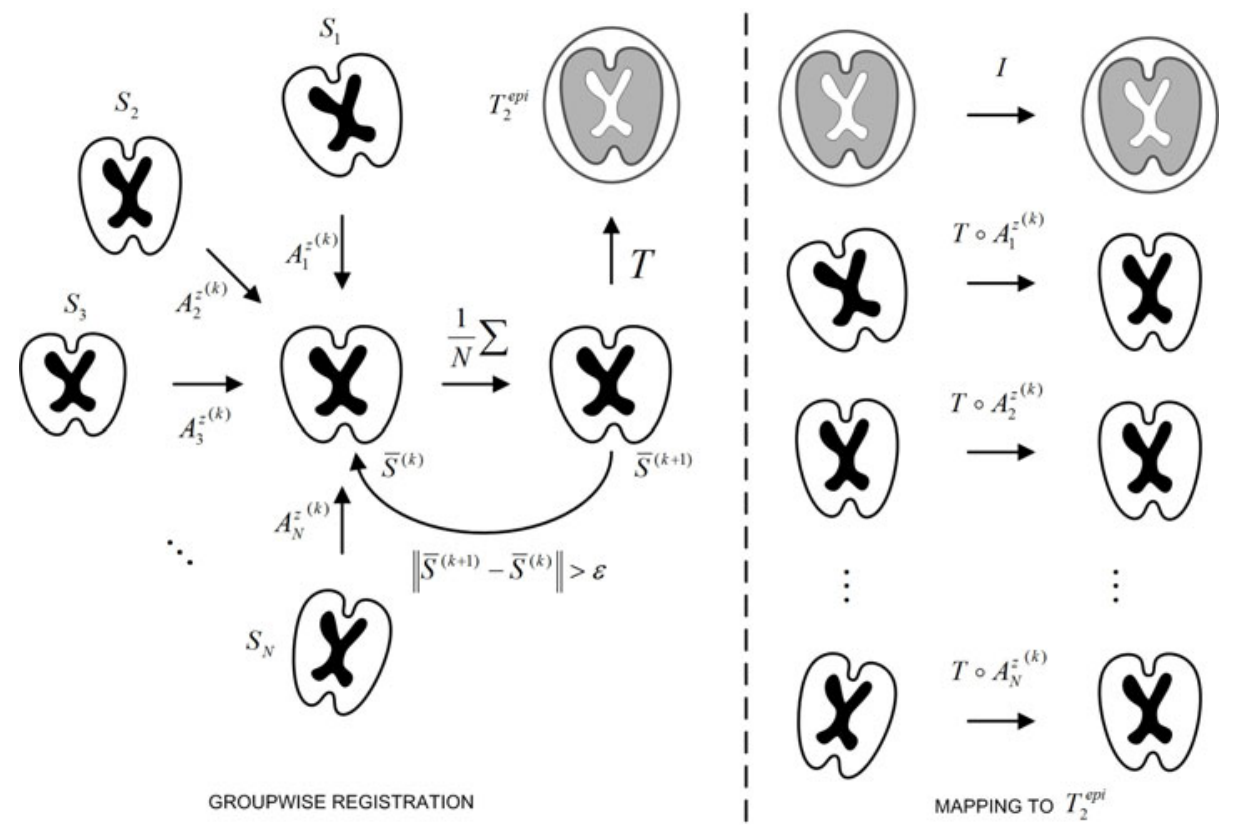

Fig. 1. Groupwise registration method. Initially all images $S_{i}$ are registered to an arbitrary DW image $S^{*}$ taken as reference. Then, all images are resampled and averaged to obtain a first average $\bar{S}^{(1)}$, which becomes the new reference. At iteration $k$, the images $S_{i}$ are registered to the mean $\bar{S}^{(k)}$ to obtain the transforms $A_{i}^{(k)}$. After convergence, $\bar{S}^{(k)}$ is registered to $T_{2}^{e p i}$, and the composition of transformations $T \circ A_{i}^{z(k)}$ is applied to map all images $S_{i}$ into the coordinate system of $T_{2}^{e p i}$.

\subsection{Sparse Interpolation}

To interpolate values on a regular grid from scattered data, we have used radial basis functions (RBF). RBFs have already been applied for interpolation on spherical geodesic grids in the context of numerical weather prediction, outperforming linear interpolation strategies [6]. The idea behind RBF interpolation is that every point has an influence on a neighborhood according to some functional $\phi(r)$, where $r$ is the distance from the point. Then, the value of the function at the point $P$ is given by a linear combination of the $\phi$ 's centered in the points $P_{i}$ :

$$
y(P)=\sum_{i=0}^{N-1} w_{i} \phi\left(\left\|P-P_{i}\right\|\right)
$$

where the weights $w_{i}$ are calculated by solving a linear system of equations for the function to agree with the observations at points $P_{i}$.

In this paper, we have used a Gaussian function as RBF since this function tends to zero for high $r$, and the influence of points $P_{i}$ distant from $P$ can be neglected. This allows considering only points in a neighborhood $\mathcal{N}(P)$ of $P$ 
for interpolation, which reduces the computational complexity of the method. $\mathcal{N}(P)$ was formed by points $P_{i}$ falling inside the support region of the Gaussian function, defined in the context of this paper as the interval $\left[-s_{\phi},+s_{\phi}\right]$ so that $\phi\left(s_{\phi}\right)=0.01 \times \phi(0)$.

In our case, each point contains spatial and angular coordinates which must be considered separately because of the difference in scale between both types of coordinates. This situation is different from the problem dealt in [6] where only an interpolation in the sphere is required. To take into account these differences, we propose to modify Equation 1 by replacing the single RBF with the product of an spatial $(\phi)$ and an angular $(\psi) \mathrm{RBF}$ :

$$
y((X, \Theta))=\sum_{i=0}^{N-1} w_{i} \phi\left(\left|X-X_{i}\right|\right) \psi\left(\left|\Theta-\Theta_{i}\right|\right)
$$

where $X=(x, y, z)$ are the spatial coordinates, and $\Theta=(\phi, \theta)$ the spherical coordinates of the sampling vector $U_{i}$. Differently from Equation 1, Equation 2 allows a dual interpolation in two different unrelated spaces. In Equation 2, $\left|X-X_{i}\right|$ represents the Euclidean distance, whereas $\left|\Theta-\Theta_{i}\right|$ is the geodesic distance over the unit sphere.

\section{Materials and Experiments}

\subsection{Image Data}

Fetal MRI was performed on a 1.5 T Siemens Avanto MRI Scanner (SIEMENS, Erlangen, Germany) at the Hautepierre Hospital (Strasbourg, France) using an 6-channel phased array coil combined to the spine array positioned around the mother abdomen. An axial spin echo single-shot echo-planar sequence was acquired along 30 non-collinear diffusion gradient encoding directions with a $b$ value of $700 \mathrm{~s} / \mathrm{mm}^{2}$. The following pulse sequence parameters were used: $\mathrm{TR}=6800 \mathrm{~ms}$; $\mathrm{TE}=99 \mathrm{~ms} ; \mathrm{FOV}=250 \times 250 \mathrm{~mm}^{2} ;$ matrix $=128 \times 128 ; 41$ contiguous axial slices of $3.5 \mathrm{~mm}$ thickness covering the whole fetal brain; no gap; number of excitations $=2$. The total imaging time was $7 \mathrm{mi} 10 \mathrm{~s}$. Pregnant women were briefed before the exam and signed informed consent. To reduce motion artifacts, fetal sedation was obtained with $1 \mathrm{mg}$ of flunitrazepam given orally to the mother 30 mi before the exam. The study was approved by the local ethics committee.

For validation purposes, three D-MRI sequences of the brain were acquired for an adult healthy subject in the following conditions: (i) static in supine position ( $\mathcal{S}^{\text {ref }}$, the reference), and (ii) static with the head rotated with respect to the reference $\left(\mathcal{S}^{\text {rot }}\right)$.

\subsection{Slice to Volume Registration Accuracy}

Initially we want to explore the ability to recover slice to volume alignment for typical but known motion, on typical anatomical structures. Fetal data are not suitable for assessing accuracy since motion artifacts are always present to some 
degree. Therefore only the adult dataset $\mathcal{S}^{\text {ref }}$ was used to this aim. Central slices of the volume were modified with a specific transformation and then registered independently to the whole volume. The displacements were chosen from a uniform distribution with a varying range of $[-8,+8] \mathrm{mm}$ for translations in each direction, and between $[-10,+10]^{\circ}$ for each rotation. These ranges of variation represent movements much larger than those observed in real fetal data.

The accuracy was assessed by computing a registration error measured on a set of 4 points $P_{i}$ within every slices as follows: $R M S=\left(\frac{1}{N} \sum_{i=1}^{N} T R E_{i}\right)^{\frac{1}{2}}$, where TRE is the target registration error defined as $T R E=\left\|P_{i}-\hat{T}^{-1}\left(T^{*}\left(P_{i}\right)\right)\right\|^{2}$. $T^{*}$ denotes the known applied motion transformation, and $\hat{T}$ is the estimated geometric transformation. $P_{i}$ are the corners of the intersection between the bounding box containing the brain, and each slice. The error previously defined provides thus an upper bound of the registration error for the region of interest.

\subsection{Evaluation of RBF Interpolation}

Leave-one-out test. A leave-one-out test by using the adult data $\mathcal{S}^{\text {ref }}$ was performed for evaluating the capability of recovering non-acquired DW images from the available measurements. This test estimates the image $S_{j}^{r e f}$ at a point $\left(X_{j}, \Theta_{j}\right)$ from $\mathcal{S}^{\text {ref }} \backslash S_{j}^{r e f}$ and $\mathcal{N}\left(X_{j}\right) \backslash X_{j}$, which in terms of RBF interpolation can be expressed as:

$$
\hat{\mathcal{S}}^{r e f}\left(X_{j}, \Theta_{j}\right)=\sum_{i=0, i \neq j}^{N-1} w_{i} \phi\left(\left|X_{j}-X_{i}\right|\right) \psi\left(\left|\Theta_{j}-\Theta_{i}\right|\right)
$$

We have then computed the RMS error between $\hat{\mathcal{S}}^{\text {ref }}\left(X_{j}, \Theta_{j}\right)$ and $\mathcal{S}^{r e f}\left(X_{j}, \Theta_{j}\right)$ for a set of random points distributed uniformly over the brain.

DW image estimation from a rotated sequence. In this experiment, DW images of $\mathcal{S}^{r e f}$ were estimated from $\mathcal{S}^{\text {rot }}$ after performing a registration to put $\mathcal{S}^{\text {rot }}$ into the frame of reference of $\mathcal{S}^{\text {ref }}$. Differently from the leave-one-out experiment, here all DW images of $\mathcal{S}^{r o t}$ at all spatial positions were employed for estimation.

\subsection{Diffusion Descriptors}

To evaluate the performance of the registration method, we have also considered two criteria for quality assessment of the resulting FA image.

Mean FA. The average value of FA over the cerebrospinal fluid (CSF), $\overline{F A}_{c f s}$ is expected to be close to zero because of the isotropic diffusion properties of the CSF. Registration errors may induce an increase of this measure, since voxels belonging to the CSF in some DW images may be matched with voxels belonging to the gray or white matter in others. 
Entropy. Nielsen et al. [7] have compared polynomial and affine distortion correction, and observed a reduction in erroneous regions of FA maps along with a more spiky FA distribution in favor of the polynomial registration. Netsch and van Muiswinkel [8] have reported an increase in the sharpness of FA maps after distortion correction. These observations correspond to lower $H_{F A}$ values.

\section{Results}

The slice-to-volume test was applied to $S_{0}^{\text {ref }}$ and $S_{i}^{\text {ref }}$. RMS values higher than $0.2 \mathrm{~mm}$ were considered as registration failures, and discarded for analysis. Under this criterion, successful registrations were obtained in $95 \%$ of the applied transformations. The obtained RMS errors were $0.162 \pm 0.004 \mathrm{~mm}$ for $S_{0}^{\text {ref }}$, and $0.105 \pm 0.011 \mathrm{~mm}$ for $S_{i}^{r e f}$. Figure 2 (a) shows this error distribution.

Th RMS error between the original sequence $\mathcal{S}^{\text {ref }}$ and its leave-one-out estimation $\hat{\mathcal{S}}^{\text {ref }}$ was lower than 0.001 for $s_{\phi} \in[2.0,4.5] \mathrm{mm}$, and $s_{\psi} \in[0.6,0.9] \mathrm{rad}$. The minimum values were obtained for $s_{\phi}=3.5 \mathrm{~mm}$ (the inter-plane resolution), suggesting that the use of only in - plane neighbors is not sufficient for interpolation purposes, and adjacent slices must be included. Higher values of $s_{\phi}$ make the interpolated value dependent of remote regions which could not present the same diffusion properties, and the error starts increasing. No dependence of $s_{\psi}$ was found in this experiment.

Figure 2 (b) shows the results when estimating $\mathcal{S}^{\text {ref }}$ from $\mathcal{S}^{\text {rot }}$. In this case the error does depends on $s_{\psi}$, being lower for higher $s_{\psi}$ values. After a given value ( 0.72 in our case) the error starts increasing again, because of the influence of distant gradient directions.

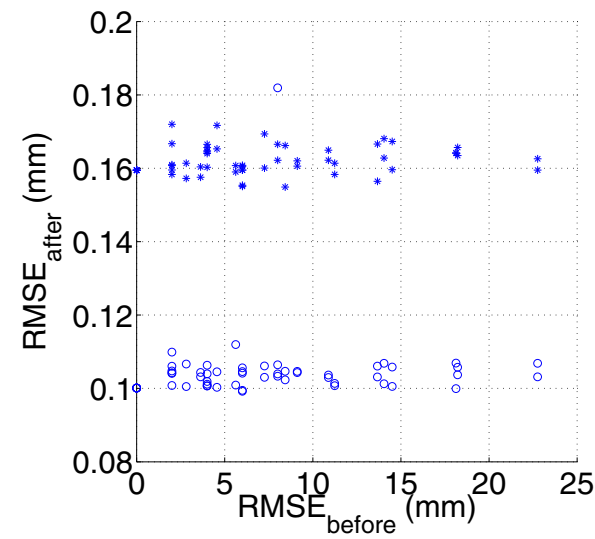

(a)

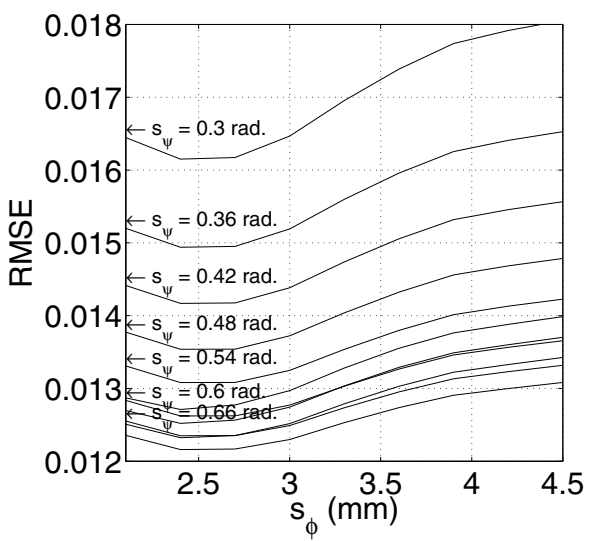

(b)

Fig. 2. (a) Slice to volume registration accuracy. This Figure shows the RMS error before and after registration for $S_{i}(\mathrm{o})$ and $T_{2}^{e p i}(+)$. Each point corresponds to a specific applied transformation. (b) Estimation of $\mathcal{S}^{\text {ref }}$ from $\mathcal{S}^{\text {rot }}$. The figure shows the normalized RMS error for different support regions of the RBF functions $\phi$ and $\psi$. 
Table 1. Diffusion descriptors based on FA maps. $H_{F A}=$ Entropy of FA, $\overline{F A}_{c s f}=$ mean FA in the CSF. For each column, the best value is shown in bold.

\begin{tabular}{|c|c|c|c|c|}
\hline$\overline{\mathrm{Met}}$ & $\begin{array}{l}\text { Fetus \#1 } \\
\text { HFA }_{\mathrm{FA}} \overline{\mathbf{F A}}_{\mathrm{csf}}\end{array}$ & $\begin{array}{l}\text { Fetus \#2 } \\
\text { HFA }_{\text {FA }} \overline{\text { FA }}_{\text {csf }}\end{array}$ & $\begin{array}{l}\text { Fetus \#3 } \\
\text { HFA }_{\text {FA }} \overline{\text { csf }}\end{array}$ & $\begin{array}{l}\text { Fetus \#4 } \\
\text { HFA }_{\text {FA }} \overline{\mathbf{F A f}}_{\mathrm{csf}}\end{array}$ \\
\hline & $\begin{array}{ll}2.04 & 0.14\end{array}$ & $\begin{array}{ll}2.17 & 0.26\end{array}$ & $2.15 \quad 0.23$ & 0.24 \\
\hline & 0.12 & 0.21 & 2.20 & 2.33 \\
\hline Our & 1.930 .08 & 1.780 .13 & 1.720 .16 & 2.200 .10 \\
\hline
\end{tabular}

Table 1 compares the values of $\overline{F A}_{c f s}$ and $\overline{F A}_{c f s}$ for the original sequences, the values provided by the scanner's manufacturer, and after applying the reconstruction method presented in this paper.

To perform the tractography, an expert radiologist traced regions containing the following bundles on $T_{2}^{s e}$ images: (i) corpus callosum, (ii) pyramidal tract, and (iii) Middle cerebellar peduncle. These regions were used for seeding the tractography after propagation to the $T_{2}^{e p i}$ image by using affine registration, and to check the presence of specific bundles. Tensor was estimated by using a standard least squares method, and the tractography was performed by applying a streamline method. In both cases we have used the algorithms implemented in Slicer1. Figure 3 shows an example of the obtained results.

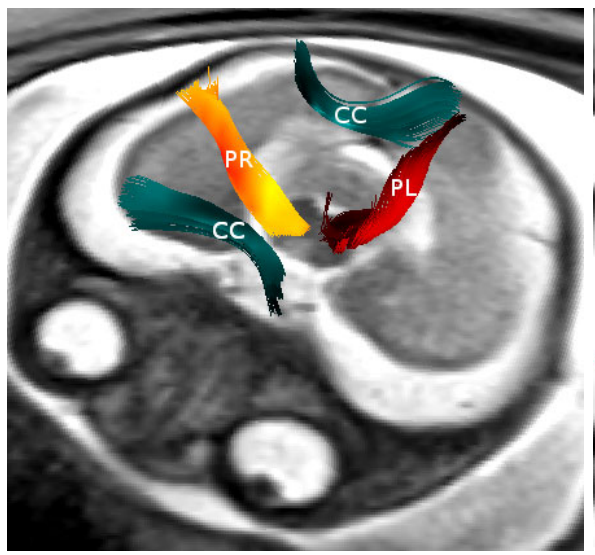

(a)

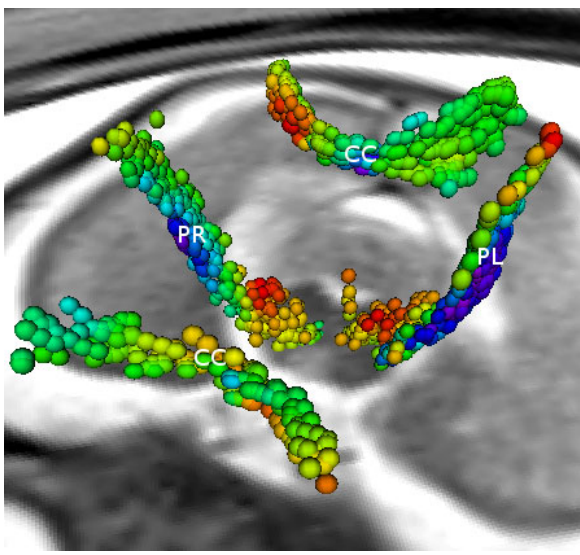

(b)

Fig. 3. Tractographies performed on a sequence corresponding to a fetus of 28 weeks of gestational age showing the corpus callosum (CC), the right pyramidal tract $(\mathrm{PR})$, and the left pyramidal tract (PL). (a) Fibers. (b) Tensors.

$\overline{1}$ http://www.slicer.org 


\section{Discussion and Conclusions}

In this paper, we have presented a method for reconstructing fetal D-MRI sequences from sparse data. A groupwise registration method based on slice-byslice affine transformations was applied to compensate motion and eddy-current distortions, and a dual spatio-angular interpolation based on RBFs was used to estimate signal values on regular sampling grids. As the proposed method does not assume any diffusion model, the generated data can be used to study diffusion patterns even in IVOH. Experiments with adult data showed a high accuracy for the slice-to-volume registration, and for the estimation of DW images along unavailable gradient directions. In fetuses, the method improved the quality of the sequences as evidenced by the lower values of $\overline{F A}_{c f s}$ and $H_{F A}$ with respect to the original ones. The tractography provided different results for the original and reconstructed sequences, but they must be quantified and compared with objective criteria in order to assess their clinical significance.

\section{Acknowledgements}

The research leading to these results has received funding from the European Research Council under the European Community's Seventh Framework Programme (FP7/2007-2013 Grant Agreement no. 207667). This work is also funded by NIH Grant R01 NS055064 and a CNRS grant for collaboration between LSIIT and BICG.

\section{References}

1. Rohde, G.K., Barnett, A.S., Basser, P.J., Marenco, S., Pierpaoli, C.: Comprehensive approach for correction of motion and distortion in diffusion-weighted MRI. Magn. Reson. Med. 51(1), 103-114 (2004)

2. Rousseau, F., Glenn, O.A., Iordanova, B., Rodriguez-Carranza, C., Vigneron, D.B., Barkovich, J.A., Studholme, C.: Registration-based approach for reconstruction of high-resolution in utero fetal MR brain images. Acad. Radiol. 13(9), 1072-1081 (2006)

3. Jiang, S., Xue, H., Counsell, S., Anjari, M., Allsop, J., Rutherford, M., Rueckert, D., Hajnal, J.V.: Diffusion tensor imaging (DTI) of the brain in moving subjects: application to in-utero fetal and ex-utero studies. Magn. Reson. Med. 62(3), 645-655 (2009)

4. Tuch, D.S., Reese, T.G., Wiegell, M.R., Makris, N., Belliveau, J.W., Wedeen, V.J.: High angular resolution diffusion imaging reveals intravoxel white matter fiber heterogeneity. Magn. Reson. Med. 48(4), 577-582 (2002)

5. Guimond, A., Meunier, J., Thirion, J.-P.: Average brain models: a convergence study. Comput. Vis. Image Understand 77, 192-210 (2000)

6. Carfora, M.F.: Interpolation on spherical geodesic grids: a comparative study. J. Comput. Appl. Math. 210(1-2), 99-105 (2007)

7. Nielsen, J.F., Ghugre, N.R., Panigrahy, A.: Affine and polynomial mutual information coregistration for artifact elimination in diffusion tensor imaging of newborns. Magn. Reson Imaging 22(9), 1319-1323 (2004)

8. Netsch, T., van Muiswinkel, A.: Quantitative evaluation of image-based distortion correction in diffusion tensor imaging. IEEE Trans. Med. Imaging 23(7), 789-798 (2004) 